\title{
A GENERALIZATION OF PILLEN'S THEOREM FOR PRINCIPAL SERIES MODULES
}

\author{
YUTAKA YOSHII \\ (Communicated by Pham Huu Tiep)
}

\begin{abstract}
Let $G$ be a connected, semisimple and simply connected algebraic group defined and split over the finite field of order $p$. Pillen proved in 1997 that the highest weight vectors of some Weyl $G$-modules generate the principal series modules as submodules for the corresponding finite Chevalley groups. This result is generalized in this paper.
\end{abstract}

\section{INTRODUCTION AND NOTATION}

Let $G$ be a connected, semisimple and simply connected algebraic group over an algebraically closed field $k$ of characteristic $p>0$ which is defined and split over the finite field $\mathbb{F}_{p}$ of order $p$. Let $G(n)$ be the corresponding finite Chevalley group consisting of the set of fixed points of the $n$-th Frobenius map $F^{n}$, and set $q=p^{n}$.

In representation theory of finite groups of Lie type (in the defining characteristic), it is interesting to investigate induced $G(n)$-modules from one-dimensional modules for a Borel subgroup of $G(n)$, which are called principal series modules. These modules have been studied by a lot of people, such as Richen, Carter, Lusztig, Jantzen, Sawada, Pillen and so on (for example, see [2], 6], 10, [1], [12]). Here we pay attention to the fact that Pillen has given a necessary and sufficient condition for the highest weight vector of a Weyl module to generate a principal series module:

Theorem 1.1 (10, Theorem 1.2]). Suppose that $q>2 h-1$ where $h$ is the Coxeter number of $G$ and suppose that $\lambda$ is a q-restricted weight. Then the highest weight vector of the Weyl module $V((q-1) \rho+\lambda)$ generates $M_{n}(\lambda)$ as a $k G(n)$-submodule if and only if $\left\langle\lambda, \alpha^{\vee}\right\rangle>0$ for all simple roots $\alpha$.

The purpose of this paper is to generalize this result. More concretely, since the latter condition $\left\langle\lambda, \alpha^{\vee}\right\rangle>0$ is somewhat strong, we would like to obtain a similar statement without the condition. The result is given in Theorem 2.1, where we see that for any $q$-restricted weight $\lambda$, the highest weight vector of $V((q-1) \rho+$ $\lambda$ ) generates a $k G(n)$-submodule which is a direct summand of $M_{n}(\lambda)$, and its indecomposable summands are completely determined. The argument is essentially similar to Pillen's original proof, but, actually, there is an error in the proof of Lemma 1.5 in [10. However, it is not so difficult to modify it. Moreover, we can also weaken the assumption on $q$ from $q>2 h-1$ to $q>h+1$.

Let $T$ be a split maximal torus of $G$. Let $B$ be an $F^{n}$-stable Borel subgroup of $G$ containing $T$, and let $B^{+}$be the opposite Borel subgroup. By $B^{+}(n)$ we denote

Received by the editors April 29, 2011.

2010 Mathematics Subject Classification. Primary 20C33. 
the corresponding finite subgroup of $B^{+}$. Let $W=N_{G}(T) / T$ be the Weyl group of $G$, and let $w_{0}$ be the longest element of $W$. Let $X=X(T)$ be the character group of $T$, and let $\Phi$ be a root system with respect to $G$ and $T$. Let $\Phi^{+}$be the set of positive roots which corresponds to the Borel subgroup $B^{+}$, and let $\Delta$ be the set of simple roots. By $\rho$ we denote the half sum of all positive roots. The Weyl group $W$ acts on $X$ in a natural way, and by using this we also can define another action (called dot action) as $w \cdot \lambda=w(\lambda+\rho)-\rho$. We can define a $W$-invariant inner product $\langle\cdot, \cdot\rangle$ on the Euclidean space $\mathbb{E}=X \otimes_{\mathbb{Z}} \mathbb{R}$. For each root $\alpha \in \Phi$, we call $\alpha^{\vee}=2 \alpha /\langle\alpha, \alpha\rangle$ a coroot of $\alpha$. Set

$$
\begin{aligned}
& X^{+}=\left\{\lambda \in X \mid\left\langle\lambda, \alpha^{\vee}\right\rangle \geq 0, \forall \alpha \in \Delta\right\}, \\
& X_{n}=\left\{\lambda \in X^{+} \mid\left\langle\lambda, \alpha^{\vee}\right\rangle<q, \forall \alpha \in \Delta\right\} .
\end{aligned}
$$

The elements of $X^{+}$and $X_{n}$ are called dominant weights and $q$-restricted weights respectively. If $\alpha_{0}$ is the highest short root, we call $h=\left\langle\rho, \alpha_{0}^{\vee}\right\rangle+1$ the Coxeter number. The dual basis (relative to the inner product) of the basis $\left\{\alpha^{\vee} \mid \alpha \in \Delta\right\}$ is denoted by $\left\{\omega_{\alpha} \mid \alpha \in \Delta\right\}$ (namely, $\left\langle\omega_{\alpha}, \beta^{\vee}\right\rangle=\delta_{\alpha, \beta}$ for any $\alpha, \beta \in \Delta$ ), and these elements are called fundamental weights. The sum of all fundamental weights is equal to $\rho$. We define an order relation on $X: \lambda \leq \mu$ if $\mu-\lambda \in \sum_{\alpha \in \Delta} \mathbb{Z}_{\geq 0} \alpha$.

By $G$-modules we mean finite-dimensional rational left $G$-modules. Let $k_{\lambda}$ be the one-dimensional $T$-module with weight $\lambda$ which can be regarded as the $B$-or $B^{+}$-module naturally. For each $\lambda \in X^{+}$, let $L(\lambda)$ be the simple $G$-module of highest weight $\lambda$, and let $V(\lambda)$ and $H^{0}(\lambda)=\operatorname{Ind}_{B}^{G} k_{\lambda}$ be the Weyl and the dual Weyl modules of highest weight $\lambda$ respectively. If $\lambda=(q-1) \rho$, we write $\mathrm{St}_{n}$ instead of $L((q-1) \rho)$ and call it the ( $n$-th) Steinberg module. Moreover, for each $\lambda \in X$, let $M_{n}(\lambda)$ be the principal series module $\operatorname{Ind}_{B^{+}(n)}^{G(n)} k_{\lambda}=k G(n) \otimes_{k B^{+}(n)} k_{\lambda}$.

\section{MAIN RESUlt}

Before stating our main theorem, we have to introduce further notation.

For a subset $I \subseteq \Delta$, let $\rho_{I}$ denote the sum of all $\omega_{\alpha}$ with $\alpha \in I$. For $\lambda \in X_{n}$, set

$$
I_{0}(\lambda)=\left\{\alpha \in \Delta \mid\left\langle\lambda, \alpha^{\vee}\right\rangle=0\right\}
$$

and

Then [6, $4.6(1)]$ says that

$$
I_{q-1}(\lambda)=\left\{\alpha \in \Delta \mid\left\langle\lambda, \alpha^{\vee}\right\rangle=q-1\right\} .
$$

$$
M_{n}(\lambda) / \operatorname{rad} M_{n}(\lambda) \cong \bigoplus_{J \subseteq I_{0}(\lambda)} \bigoplus_{J^{\prime} \subseteq I_{q-1}(\lambda)} L\left(\lambda+(q-1) \rho_{J}-(q-1) \rho_{J^{\prime}}\right) .
$$

On the other hand, it is well known that the induced module $\operatorname{Ind}_{U^{+}(n)}^{G(n)} k$ can be decomposed as

$$
\operatorname{Ind}_{U^{+}(n)}^{G(n)} k=\bigoplus_{\lambda \in X_{n}} Y(\lambda)
$$

where each indecomposable summand $Y(\lambda)$ has a simple head $L(\lambda)$ (see [12, Section 3]). Since the induced $B^{+}(n)$-module $\operatorname{Ind}_{U^{+}(n)}^{B^{+}(n)} k$ is semisimple, it follows that $M_{n}(\lambda)$ is a direct summand of $\operatorname{Ind}_{U^{+}(n)}^{G(n)} k$, and we can write

$$
M_{n}(\lambda)=\bigoplus_{J \subseteq I_{0}(\lambda)} \bigoplus_{J^{\prime} \subseteq I_{q-1}(\lambda)} Y\left(\lambda+(q-1) \rho_{J}-(q-1) \rho_{J^{\prime}}\right) .
$$


For a subset $I \subseteq \Delta$, we denote its complement by $I^{c}$. Let $G_{I}$ be the Levi subgroup relative to $I$, and let $L_{I}(\lambda), V_{I}(\lambda)$ and $H_{I}^{0}(\lambda)$ be the simple, the Weyl and the dual Weyl $G_{I}$-modules with highest weight $\lambda$ respectively. Let $G_{I}(n)$ be the corresponding finite group of $G_{I}$ and set $M_{n, I}(\lambda)=\operatorname{Ind}_{B_{I}^{+}(n)}^{G_{I}(n)} k_{\lambda}$, where $B_{I}^{+}=G_{I} \cap B^{+}$and $B_{I}^{+}(n)$ is the corresponding finite group of $B_{I}^{+}$. As in the case of $G$, we write $Y_{I}(\mu)$ for the indecomposable summand of $M_{n, I}(\lambda)$ with simple head $L_{I}(\mu)$.

Now we state our main theorem.

Theorem 2.1. Let $\lambda \in X_{n}$, and suppose that $\left.q\right\rangle\left\langle\rho_{I_{0}(\lambda)^{c}}, \alpha_{0}^{\vee}\right\rangle+2$. Then the $G(n)$ submodule generated by the highest weight vector of $V((q-1) \rho+\lambda)$ is isomorphic to

$$
\bigoplus_{J \subseteq I_{q-1}(\lambda)} Y\left(\lambda+(q-1) \rho_{I_{0}(\lambda)}-(q-1) \rho_{J}\right)
$$

Note that the theorem holds for all $\lambda \in X_{n}$ if $q>h+1$ and that the above direct sum is isomorphic to $M_{n}(\lambda)$ if $I_{0}(\lambda)$ is empty. Therefore, this theorem is a generalization of Theorem 1.1 .

The proof will involve a series of lemmas.

Lemma 2.2 ([10, Lemma 1.3]). Let $\lambda \in X_{n}$ and $\nu \in X^{+}$. Let $m_{\lambda}$ be a generator of the $G(n)$-module $M_{n}(\lambda)$ and let $v_{\nu}$ be the highest weight vector of $V(\nu)$. Then $M_{n}(\lambda) \otimes V(\nu)$ contains $M_{n}(\lambda+\nu)$ as the $G(n)$-submodule generated by $m_{\lambda} \otimes v_{\nu}$.

Lemma 2.3 ([10, Lemma 1.4]). Let $\lambda, \mu \in X_{n}$. If the simple $G(n)$-module $L(\mu)$ is a composition factor of $\operatorname{soc}_{G(n)}\left(\mathrm{St}_{n} \otimes V(\lambda)\right)$, then $\mu \geq(q-1) \rho+\lambda$.

For the next lemmas, we have to introduce some notation. Let $G_{n}$ be the (scheme-theoretic) kernel of the $n$-th Frobenius map. For $\lambda \in X, \widehat{L}_{n}(\lambda)$ denotes the simple $G_{n} T$-module with highest weight $\lambda$, and $\widehat{Q}_{n}(\lambda)$ denotes the injective hull (= projective cover) of $\widehat{L}_{n}(\lambda)$. Let $\widehat{Z}_{n}(\lambda)$ be the coinduced $G_{n} T$-module with highest weight $\lambda$ (see [7, II, 9.1 (2)]). If $H$ is a group and if $V$ and $L$ are $H$-modules with $L$ simple, then we denote by $[V: L]_{H}$ the multiplicity of $L$ in the composition factors of $V$.

The following two lemmas are generalizations of Lemmas 1.5 and 1.6 in [10]. Actually, Pillen's proof of Lemma 1.5 is not complete. (Indeed, he asserts there that $2 \rho \geq(q-1) \nu \geq 0$ implies $2(h-1) \geq(q-1)\left\langle\nu, \alpha^{\vee}\right\rangle \geq 0$ for all $\alpha \in \Delta$, but this is clearly not true.) However, the modification is not so difficult, and moreover the modified proof requires a weaker assumption on $q$ than $q>2 h-1$.

Lemma 2.4. Let $I \subseteq \Delta$, and suppose that $q>\left\langle\rho_{I}, \alpha_{0}^{\vee}\right\rangle+2$. If $\mu \in X_{n}$ satisfies $\mu \geq(q-1) \rho+w_{0} \rho_{I}$, then we have

$$
\left[M_{n}\left(\rho_{I}\right): L(\mu)\right]_{G(n)}= \begin{cases}1 & \text { if } \mu=(q-1) \rho+w_{0} \rho_{I} \\ 0 & \text { if } \mu>(q-1) \rho+w_{0} \rho_{I}\end{cases}
$$


Proof. We have

$$
\begin{aligned}
{\left[M_{n}\left(\rho_{I}\right): L(\mu)\right]_{G(n)} } & \leq \sum_{\nu \in X}\left[\widehat{Z}_{n}\left(\rho_{I}+\nu+(q-1) \rho\right): \widehat{L}_{n}(q \nu+\mu)\right]_{G_{n} T} \\
& =\sum_{\nu \in X}\left[\widehat{Z}_{n}\left((q-1) \rho+\rho_{I}-(q-1) \nu\right): \widehat{L}_{n}(\mu)\right]_{G_{n} T} \\
& =\sum_{\nu \in X}\left[\widehat{Q}_{n}(\mu): \widehat{Z}_{n}\left((q-1) \rho+\rho_{I}-(q-1) \nu\right)\right]_{G_{n} T}
\end{aligned}
$$

where $\left[\widehat{Q}_{n}(\mu): \widehat{Z}_{n}(\nu)\right]_{G_{n} T}$ is the multiplicity of $\widehat{Z}_{n}(\nu)$ in the $\widehat{Z}_{n}$-filtration of $\widehat{Q}_{n}(\mu)$ (see [7, Proposition II, 11.4]). The first inequality follows from [9, Lemma 4.3 (3)] and [5, 3.2], and the second and the last equalities follow from [4, 2.4 (7)] and Brauer-Humphreys reciprocity. Suppose that $\widehat{L}_{n}(\mu)$ appears in the composition factors of $\widehat{Z}_{n}\left((q-1) \rho+\rho_{I}-(q-1) \nu\right)$. Then we see from [4, Satz $\left.3.2(1)\right]$ that $\widehat{L}_{n}(\mu)$ appears also in those of $\widehat{Z}_{n}\left(q \rho+w \cdot\left(\rho_{I}-\rho-(q-1) \nu\right)\right)$ for all $w \in W$. Then we have

$$
\mu \uparrow q \rho+w \cdot\left(\rho_{I}-\rho-(q-1) \nu\right) \uparrow 2(q-1) \rho+w_{0} \mu
$$

(see [7, Lemma II, 9.16.a] or [4, Satz 3.3]), where $\uparrow$ is the order relation on $X$ defined in [7, II, Chapter 6]. These inequalities and the assumption on $\mu$ imply

$$
\begin{aligned}
w \rho_{I}-\rho_{I} & \leq-(q-1) \rho-w_{0} \mu+w \rho_{I} \leq(q-1) w \nu \\
& \leq(q-1) \rho-\mu+w \rho_{I} \leq w \rho_{I}-w_{0} \rho_{I} .
\end{aligned}
$$

We shall show that $\nu=0$, and then $\mu$ must be equal to $(q-1) \rho+w_{0} \rho_{I}$ by taking $w=1$ in the last inequalities. Without loss of generality, we may assume that the root system $\Phi$ is indecomposable. Then we know that at most two root lengths occur in $\Phi$ and that all roots of a given length are $W$-conjugate (see 3 , Lemma $10.4 \mathrm{C}]$ ). Let $\alpha \in \Delta$ be any simple root, and let $\beta_{0}$ be the highest root with the same length as $\alpha$. Then there is an element $w \in W$ such that $\alpha=w \beta_{0}$. Moreover, we know that $\left\langle\lambda, \beta_{0}^{\vee}\right\rangle \leq\left\langle\lambda, \alpha_{0}^{\vee}\right\rangle$ for all $\lambda \in X^{+}$. Now we have

$$
\begin{aligned}
\left\langle w \rho_{I}-\rho_{I}, \beta_{0}^{\vee}\right\rangle & =\left\langle\rho_{I}, \alpha^{\vee}\right\rangle-\left\langle\rho_{I}, \beta_{0}^{\vee}\right\rangle \\
& >-(q-2), \\
\left\langle w \rho_{I}-w_{0} \rho_{I}, \beta_{0}^{\vee}\right\rangle & =\left\langle\rho_{I}, \alpha^{\vee}\right\rangle+\left\langle\rho_{I}, \beta_{0}^{\vee}\right\rangle \\
& <q-1,
\end{aligned}
$$

and $\left\langle(q-1) w \nu, \beta_{0}^{\vee}\right\rangle=(q-1)\left\langle\nu, \alpha^{\vee}\right\rangle$. The fact that $\left\langle\gamma, \beta_{0}^{\vee}\right\rangle \geq 0$ for all $\gamma \in \Delta$ shows $-(q-2)<(q-1)\left\langle\nu, \alpha^{\vee}\right\rangle\left\langle q-1\right.$; hence $\left\langle\nu, \alpha^{\vee}\right\rangle=0$. Since $\alpha \in \Delta$ is arbitrary, we have $\nu=0$.

It remains to check that $\left[M_{n}\left(\rho_{I}\right): L\left((q-1) \rho+w_{0} \rho_{I}\right)\right]_{G(n)}=1$. Now we know that the multiplicity is less than or equal to the multiplicity $\left[\widehat{Q}_{n}\left((q-1) \rho+w_{0} \rho_{I}\right)\right.$ : $\left.\widehat{Z}_{n}\left((q-1) \rho+\rho_{I}\right)\right]_{G_{n} T}$, which is at most one since $(q-1) \rho+\rho_{I}$ is the highest weight of $\widehat{Q}_{n}\left((q-1) \rho+w_{0} \rho_{I}\right)$ with multiplicity one. On the other hand, by [6, $\left.4.6(2)\right]$, $L\left((q-1) \rho+w_{0} \rho_{I}\right)$ appears in the composition factors of the socle of $M_{n}\left(\rho_{I}\right)$, and the lemma follows.

Remark. Even if $q$ does not satisfy the assumption, the lemma holds if the pair $(\nu, \mu)$ in $(\mathrm{A})$ can be only $\left(0,(q-1) \rho+w_{0} \rho_{I}\right)$. But unfortunately, the lemma does not always hold. For example, if $q=3, I=\Delta$ and $\mu=(q-1) \rho+w_{0} \rho_{I}=\rho$, then 
$\left[M_{n}(\rho): L(\mu)\right]_{G(n)} \geq 2$ since $L(\rho)$ appears both in the head and in the socle of the nonsimple module $M_{n}(\rho)$. In types $A_{2}, B_{2}$ and $G_{2}$, we can check by computing the pairs $(\nu, \mu)$ directly that the lemma always holds unless $q=2$ or 3 .

Lemma 2.5. Let $I \subseteq \Delta$, and suppose that $q\rangle\left\langle\rho_{I}, \alpha_{0}^{\vee}\right\rangle+2$. Then the $G(n)$ submodule generated by the highest weight vector of $V\left((q-1) \rho+\rho_{I}\right)$ is isomorphic to $Y\left(\rho_{I}+(q-1) \rho_{I^{c}}\right)$.

Proof. Let $v$ be the highest weight vector (with weight $\left.(q-1) \rho+\rho_{I}\right)$ of the tensor product $\mathrm{St}_{\mathrm{n}} \otimes V\left(\rho_{I}\right)$. Note that this tensor product has a Weyl filtration (see 8 , Theorem 1]) and that $v$ generates a $G$-submodule isomorphic to $V\left((q-1) \rho+\rho_{I}\right)$ since the Weyl module is the only one which occurs on the bottom of any Weyl filtration of it (see [1, 1.2]).

Consider the $k G(n)$-homomorphism $f: M_{n}\left(\rho_{I}\right) \rightarrow \mathrm{St}_{n} \otimes V\left(\rho_{I}\right)$ obtained from the natural $k B^{+}(n)$-homomorphism

$$
k_{\rho_{I}}=k_{(q-1) \rho+\rho_{I}} \rightarrow \mathrm{St}_{n} \otimes V\left(\rho_{I}\right), \quad 1 \mapsto v
$$

through the Frobenius reciprocity

$$
\operatorname{Hom}_{B^{+}(n)}\left(k_{(q-1) \rho+\rho_{I}}, \mathrm{St}_{n} \otimes V\left(\rho_{I}\right)\right) \cong \operatorname{Hom}_{G(n)}\left(M_{n}\left(\rho_{I}\right), \mathrm{St}_{n} \otimes V\left(\rho_{I}\right)\right) .
$$

Note that $\operatorname{Im}(f) \neq 0$. Now Lemmas 2.3 and 2.4 imply that the $G(n)$-socle of $\operatorname{Im}(f)$ is isomorphic to $L\left((q-1) \rho+w_{0} \rho_{I}\right)$. Let $\tilde{f}$ be the composite map of the canonical inclusion $Y\left(\rho_{I}+(q-1) \rho_{I^{c}}\right) \subseteq M_{n}\left(\rho_{I}\right)$ and $f: M_{n}\left(\rho_{I}\right) \rightarrow \mathrm{St}_{n} \otimes V\left(\rho_{I}\right)$. By [12, Theorem 3.10], the socle of $Y\left(\rho_{I}+(q-1) \rho_{I^{c}}\right)$ is $L\left((q-1) \rho+w_{0} \rho_{I}\right)$. Recall that $M_{n}(\rho)$ has $L\left((q-1) \rho+w_{0} \rho_{I}\right)$ as a composition factor only in the socle, whose multiplicity is one. Hence $\tilde{f}$ must be injective, since it takes the socle of $Y\left(\rho_{I}+(q-1) \rho_{I^{c}}\right)$ into the socle of $\operatorname{Im}(f)$. On the other hand, since $\operatorname{Im}(\tilde{f})=\operatorname{Im}(f)$ is the $k G(n)$-submodule in $\mathrm{St}_{n} \otimes V\left(\rho_{I}\right)$ generated by $v$, it follows that $\operatorname{Im}(\tilde{f}) \subseteq V\left((q-1) \rho+\rho_{I}\right)$.

Remark. Since the socle of $M_{n}\left(\rho_{I}\right)$ is multiplicity-free, the above map $f$ is always injective on $Y\left(\rho_{I}+(q-1) \rho_{I^{c}}\right)$ and zero on any other summand for any indecomposable decomposition of $M_{n}\left(\rho_{I}\right)$.

Now we turn to prove our main theorem.

Proof of Theorem 2.1. We decompose $M_{n}\left(\rho_{I_{0}(\lambda)^{c}}\right)$ as

$$
M_{n}\left(\rho_{I_{0}(\lambda)^{c}}\right)=\bigoplus_{J \subseteq I_{0}(\lambda)} Y\left(\rho_{I_{0}(\lambda)^{c}}+(q-1) \rho_{J}\right)
$$

and consider the $k G(n)$-homomorphism

$$
f \otimes i d: M_{n}\left(\rho_{I_{0}(\lambda)^{c}}\right) \otimes V\left(\lambda-\rho_{I_{0}(\lambda)^{c}}\right) \rightarrow V\left((q-1) \rho+\rho_{I_{0}(\lambda)^{c}}\right) \otimes V\left(\lambda-\rho_{I_{0}(\lambda)^{c}}\right),
$$

where $f$ is the map $M_{n}\left(\rho_{I_{0}(\lambda)^{c}}\right) \rightarrow \mathrm{St}_{n} \otimes V\left(\rho_{I_{0}(\lambda)^{c}}\right)$ constructed in the proof of Lemma 2.5 (note that $\operatorname{Im}(f) \subseteq V\left((q-1) \rho+\rho_{I_{0}(\lambda)^{c}}\right)$ ). Let $v$ be the highest weight vector of $V\left(\lambda-\rho_{I_{0}(\lambda)^{c}}\right)$ and let $m \in k_{\rho_{I_{0}(\lambda)^{c}}}$ be a generator of the $k G(n)$-module $M_{n}\left(\rho_{I_{0}(\lambda)^{c}}\right)$. Observe that $f(m) \otimes v$ generates the $G$-submodule $V((q-1) \rho+\lambda)$. Therefore, it suffices to prove that the resulting $k G(n)$-module in the theorem is the image of the composite map of the embedding $\varphi: M_{n}(\lambda) \rightarrow M_{n}\left(\rho_{I_{0}(\lambda)^{c}}\right) \otimes V(\lambda-$ $\left.\rho_{I_{0}(\lambda)^{c}}\right)$ in Lemma 2.2 and $f \otimes i d$. 
Consider the $k G_{I_{0}(\lambda)}(n)$-module embedding

$$
\varphi_{I_{0}(\lambda)}: M_{n, I_{0}(\lambda)}(\lambda) \rightarrow M_{n, I_{0}(\lambda)}\left(\rho_{I_{0}(\lambda)^{c}}\right) \otimes V_{I_{0}(\lambda)}\left(\lambda-\rho_{I_{0}(\lambda)^{c}}\right)
$$

which is analogous to $\varphi$. By the argument in [7, II, 1.18], we see that the onedimensional $T$-module of weight $\lambda-\rho_{I_{0}(\lambda)^{c}}$ can be uniquely extended to a $G_{I_{0}(\lambda)}$-module and then it is trivial as a module for the derived subgroup $\left[G_{I_{0}(\lambda)}\right.$, $\left.G_{I_{0}(\lambda)}\right]$ of $G_{I_{0}(\lambda)}$. Now [7, Proposition I, 6.13 (1)] shows that the induced module $H_{I_{0}(\lambda)}^{0}\left(\lambda-\rho_{I_{0}(\lambda)^{c}}\right)$, and hence $V_{I_{0}(\lambda)}\left(\lambda-\rho_{I_{0}(\lambda)^{c}}\right)$, is the one-dimensional $G_{I_{0}(\lambda)^{-}}$ module $k_{\lambda-\rho_{I_{0}(\lambda) c}}$. By comparison of dimensions, we see that $\varphi_{I_{0}(\lambda)}$ is bijective and it takes the summand $Y_{I_{0}(\lambda)}\left(\lambda+(q-1) \rho_{J}\right)$ onto

$$
Y_{I_{0}(\lambda)}\left(\rho_{I_{0}(\lambda)^{c}}+(q-1) \rho_{J}\right) \otimes V_{I_{0}(\lambda)}\left(\lambda-\rho_{I_{0}(\lambda)^{c}}\right)
$$

for any $J \subseteq \Delta$. We denote this restriction map by $\varphi_{I_{0}(\lambda), J}$ (this is independent of the choice of indecomposable decompositions of various $M_{n}(\cdot)$ 's thanks to multiplicityfree property of the socle). Since the highest weight vector $v$ of $V\left(\lambda-\rho_{I_{0}(\lambda)^{c}}\right)$ generates the $G_{I_{0}(\lambda)}$-submodule $k v=V_{I_{0}(\lambda)}\left(\lambda-\rho_{I_{0}(\lambda)^{c}}\right)$, we can regard $\varphi_{I_{0}(\lambda)}$ and $\varphi_{I_{0}(\lambda), J}$ as injective homomorphisms

$$
\begin{gathered}
\varphi_{I_{0}(\lambda)}: M_{n, I_{0}(\lambda)}(\lambda) \rightarrow M_{n, I_{0}(\lambda)}\left(\rho_{I_{0}(\lambda)^{c}}\right) \otimes V\left(\lambda-\rho_{I_{0}(\lambda)^{c}}\right), \\
\varphi_{I_{0}(\lambda), J}: Y_{I_{0}(\lambda)}\left(\lambda+(q-1) \rho_{J}\right) \rightarrow Y_{I_{0}(\lambda)}\left(\rho_{I_{0}(\lambda)^{c}}+(q-1) \rho_{J}\right) \otimes V\left(\lambda-\rho_{I_{0}(\lambda)^{c}}\right) .
\end{gathered}
$$

Now we apply the Harish-Chandra induction $\operatorname{HCInd}_{G_{I_{0}(\lambda)}(n)}^{G(n)}$ to these maps. Clearly we have

$$
\begin{aligned}
\varphi & =\operatorname{HCInd}\left(\varphi_{I_{0}(\lambda)}\right)=\operatorname{HCInd}\left(\bigoplus_{J \subseteq I_{0}(\lambda)} \varphi_{I_{0}(\lambda), J}\right) \\
& =\bigoplus_{J \subseteq I_{0}(\lambda)} \operatorname{HCInd}\left(\varphi_{I_{0}(\lambda), J}\right) .
\end{aligned}
$$

We shall claim that

(B) $\operatorname{HCInd}_{G_{I_{0}(\lambda)}(n)}^{G(n)} Y_{I_{0}(\lambda)}\left(\lambda+(q-1) \rho_{J}\right)=\bigoplus_{J^{\prime} \subseteq I_{q-1}(\lambda)} Y\left(\lambda+(q-1) \rho_{J}-(q-1) \rho_{J^{\prime}}\right)$

and

(C) $\quad \operatorname{HCInd}_{G_{I_{0}(\lambda)}(n)}^{G(n)} Y_{I_{0}(\lambda)}\left(\rho_{I_{0}(\lambda)^{c}}+(q-1) \rho_{J}\right)=Y\left(\rho_{I_{0}(\lambda)^{c}}+(q-1) \rho_{J}\right)$.

Now since $q \neq 2,(\mathrm{C})$ is the special case of $\lambda=\rho_{I_{0}(\lambda)^{c}}$ in (B). For a subset $J^{\prime} \subseteq$ $I_{q-1}(\lambda)$, by Frobenius reciprocity we have

$$
\begin{aligned}
& \operatorname{Hom}_{G(n)}\left(\operatorname{HCInd}_{G_{I_{0}(\lambda)}(n)}^{G(n)} Y_{I_{0}(\lambda)}\left(\lambda+(q-1) \rho_{J}\right), L\left(\lambda+(q-1) \rho_{J}-(q-1) \rho_{J^{\prime}}\right)\right) \\
& \cong \operatorname{Hom}_{P_{I_{0}(\lambda)}^{+}(n)}\left(Y_{I_{0}(\lambda)}\left(\lambda+(q-1) \rho_{J}\right), L\left(\lambda+(q-1) \rho_{J}-(q-1) \rho_{J^{\prime}}\right)\right),
\end{aligned}
$$

where $P_{I_{0}(\lambda)}^{+}$is the parabolic subgroup of $G$ which corresponds to $I_{0}(\lambda)$ and contains $B^{+}$and $P_{I_{0}(\lambda)}^{+}(n)$ is the corresponding finite group. The $G$-module $L(\lambda+$ $\left.(q-1) \rho_{J}-(q-1) \rho_{J^{\prime}}\right)$ has a $P_{I_{0}(\lambda)}^{+}$-submodule isomorphic to $L_{I_{0}(\lambda)}(\lambda+$ $\left.(q-1) \rho_{J}-(q-1) \rho_{J^{\prime}}\right)$, which is isomorphic to $L_{I_{0}(\lambda)}\left(\lambda+(q-1) \rho_{J}\right)$ as a $k P_{I_{0}(\lambda)}^{+}(n)$ submodule. Hence the right-hand side of the last formula is nonzero, and so the 
direct sum $\bigoplus_{J^{\prime} \subseteq I_{q-1}(\lambda)} L\left(\lambda+(q-1) \rho_{J}-(q-1) \rho_{J^{\prime}}\right)$ must be contained in the head of $\operatorname{HCInd}_{G_{I_{0}(\lambda)}(n)}^{G(n)} Y_{I_{0}(\lambda)}\left(\lambda+(q-1) \rho_{J}\right)$. However, the $k G(n)$-module

$$
\bigoplus_{J \subseteq I_{0}(\lambda)} \bigoplus_{J^{\prime} \subseteq I_{q-1}(\lambda)} L\left(\lambda+(q-1) \rho_{J}-(q-1) \rho_{J^{\prime}}\right)
$$

fills the head of

$$
\begin{aligned}
M_{n}(\lambda) & =\operatorname{HCInd}_{G_{I_{0}(\lambda)}(n)}^{G(n)} M_{n, I_{0}(\lambda)}(\lambda) \\
& =\bigoplus_{J \subseteq I_{0}(\lambda)} \operatorname{HCInd}_{G_{I_{0}(\lambda)}(n)}^{G(n)} Y_{I_{0}(\lambda)}\left(\lambda+(q-1) \rho_{J}\right) ;
\end{aligned}
$$

hence the equality must hold in $(\mathrm{B})$.

Therefore, $\varphi$ maps $\bigoplus_{J^{\prime} \subseteq I_{q-1}(\lambda)} Y\left(\lambda+(q-1) \rho_{J}-(q-1) \rho_{J^{\prime}}\right)$ into the tensor product $Y\left(\rho_{I_{0}(\lambda)^{c}}+(q-1) \rho_{J}\right) \otimes V\left(\lambda-\rho_{I_{0}(\lambda)^{c}}\right)$ injectively. Moreover, by Lemma 2.5, the restriction of $f \otimes i d$ on the tensor product is injective for $J=I_{0}(\lambda)$ and zero otherwise. Since the image of the composite map $(f \otimes i d) \circ \varphi$ is the $k G(n)$-submodule generated by the highest weight vector, the result follows.

Even if $q \leq\left\langle\rho_{I_{0}(\lambda)^{c}}, \alpha_{0}^{\vee}\right\rangle+2$, when $q \neq 2$ and $I \subseteq \Delta$ satisfies Lemma 2.4, it is shown similarly that Theorem 2.1 holds. Hence the remark of Lemma 2.4 says that in types $A_{2}, B_{2}$ and $G_{2}$, Theorem 2.1 holds for all $\lambda \in X_{n}$ unless $q=2$ or 3 . Though the approach in this paper does not cover all $q$ and $\lambda$, the theorem is expected to hold in general:

Conjecture 2.6. If $\lambda \in X_{n}$, the $G(n)$-submodule generated by the highest weight vector of $V((q-1) \rho+\lambda)$ is isomorphic to

$$
\bigoplus_{J \subseteq I_{q-1}(\lambda)} Y\left(\lambda+(q-1) \rho_{I_{0}(\lambda)}-(q-1) \rho_{J}\right)
$$

\section{ACKNOWLEDGEMENT}

The author would like to thank Professor Shigeo Koshitani for carefully reading this article and giving him a lot of advice.

\section{REFERENCES}

[1] H. H. Andersen, A sum formula for tilting modules, J. Pure and Applied Algebra 152 (2000), 17-40. MR1783982 (2001g:20057)

[2] R.W. Carter and G. Lusztig, Modular representations of finite groups of Lie type, Proc. London Math. Soc. 32 (1976), 347-384. MR0396731(53:592)

[3] J. E. Humphreys, Introduction to Lie Algebras and Representation Theory, Grad. Texts Math. 9, Springer, 1972. MR0323842 (48:2197)

[4] J. C. Jantzen, Darstellungen halbeinfacher Gruppen und ihrer Frobenius-Kerne, J. Reine Angew. Math. 317 (1980), 157-199. MR581341 (82b:20057)

[5] J. C. Jantzen, Zur Reduktion modulo $p$ der Charaktere von Deligne und Lusztig, J. Algebra 70 (1981), 452-474. MR623819 (82m:20045)

[6] J. C. Jantzen, Filtrierungen der Darstellungen in der Hauptserie endlicher ChevalleyGruppen, Proc. London Math. Soc. (3) 49 (1984), 445-482. MR759299 (86e:20050)

[7] J. C. Jantzen, Representations of Algebraic Groups, 2nd ed., Math. Surveys Monogr. 107, Amer. Math. Soc., 2003. MR2015057(2004h:20061) 
[8] O. Mathieu, Filtrations of G-modules, Ann. Sci. Éc. Norm. Super., II. Ser. 23 (1990), 625-644. MR $1072820(92 \mathrm{a}: 20044)$

[9] C. Pillen, Reduction modulo $p$ of some Deligne-Lusztig characters, Arch. Math. 61 (1993), 421-433. MR 1241047 (94i:20026)

[10] C. Pillen, Loewy series for principal series representations of finite Chevalley groups, J. Algebra 189 (1997), 101-124. MR1432367(98a:20019)

[11] F.A. Richen, Modular representations of split $B N$-pairs, Trans. Amer. Math. Soc. 140 (1969), 435-460. MR0242972(39:4299)

[12] H. Sawada, A characterization of the modular representations of finite groups with split $(B, N)$-pairs, Math. Z. 155 (1977), 29-41. MR0450384(56:8679)

Department of Liberal Studies, Nara National College of Technology, YamatokoRIYAMA, NARA, 639-1080, JAPAN

E-mail address: yyoshii@libe.nara-k.ac.jp 